\title{
Effective Chemical Processes in Porous Media
}

\author{
C. Conca, J. I. Díaz, C. Timofte
}

\begin{abstract}
In the book by U. HoRNUNG, Chapter 6 , the author proposes an homogenization strategy for the effective behavior of some chemical processes involving adsorption and reactions arising in porous media. Rigorous proofs of the convergence results are given in the case of linear adsorption rates and linear chemical reactions. The author leaves as an open question the case of a nonlinear adsorption rate. Our goal in this paper is to study two well-known examples of such nonlinear models, namely the so-called Freundlich and Langmuir kinetics.
\end{abstract}

Key words: nonlinear reaction-diffusion coupling, reactive flows, adsorption.

\section{Introduction}

The general question which will make the object of this paper is the effective behavior of chemical reactive flows involving diffusion, different types of adsorption rates and chemical reactions which take place at the boundary of a periodically perforated material. For a nice presentation of the chemical aspects involved in our model (and also for some mathematical and historical backgrounds) we refer to S.N. Antontsev et Al. [1], J. Bear [4], J.I. Díaz [14], [15], J.I. Díaz and I. STAKGOLD [16] and U. HoRnUng [18].

Let $\Omega$ be an open bounded set in $\mathbb{R}^{n}$ and let us perforate it by holes. As a result, we obtain an open set $\Omega^{\varepsilon}$ which will be referred to as being the perforated domain ; $\varepsilon$ represents a small parameter related to the characteristic size of the perforations.

The nonlinear problem studied in this paper concerns the asymptotic behavior, as $\varepsilon \rightarrow 0$, of the microscopic models $\left(V^{\varepsilon}\right)$ and $\left(W^{\varepsilon}\right)$ below. In the chemical situation behind this model the domain $\Omega$ consists of two parts: a fluid phase $\Omega^{\varepsilon}$ and a solid skeleton (grains or pores), $\Omega \backslash \bar{\Omega}^{\varepsilon}$. We assume that chemical substances are dissolved in the fluid part $\Omega^{\varepsilon}$. They are transported by diffusion and also, by adsorption, they can change from being dissolved in the fluid to residing on the surface of the pores. Here, on the boundary, chemical reactions (which can be influenced by catalysts) take place. Hence, the model consists of a diffusion system in the fluid phase $\Omega^{\varepsilon}$, a reaction system on the pore surface and a boundary condition coupling them (see (1.2)). A simplified modelling of this situation is as follows:

$$
\begin{aligned}
& \left(V^{\varepsilon}\right)\left\{\begin{array}{l}
\frac{\partial v^{\varepsilon}}{\partial t}(t, x)-D \Delta v^{\varepsilon}(t, x)=h(t, x), \quad x \in \Omega^{\varepsilon}, t>0 \\
v^{\varepsilon}(t, x)=0, \quad x \in \partial \Omega, t>0 \\
v^{\varepsilon}(t, x)=v_{1}(x), \quad x \in \Omega^{\varepsilon}, t=0
\end{array}\right. \\
& \quad-D \frac{\partial v^{\varepsilon}}{\partial \nu}(t, x)=\varepsilon f^{\varepsilon}(t, x), \quad x \in S^{\varepsilon}, t>0
\end{aligned}
$$


and

$$
\left(W^{\varepsilon}\right)\left\{\begin{array}{l}
\frac{\partial w^{\varepsilon}}{\partial t}(t, x)+a w^{\varepsilon}(t, x)=f^{\varepsilon}(t, x), \quad x \in S^{\varepsilon}, t>0 \\
w^{\varepsilon}(t, x)=w_{1}(x), \quad x \in S^{\varepsilon}, t=0
\end{array}\right.
$$

where

$$
f^{\varepsilon}(t, x)=\gamma\left(g\left(v^{\varepsilon}(t, x)\right)-w^{\varepsilon}(t, x)\right) .
$$

Here, $\nu$ is the exterior unit normal to $\Omega^{\varepsilon}, a, \gamma>0, h$ is a given function representing an external source of energy, $v_{1}, w_{1} \in H_{0}^{1}(\Omega)$ and $S^{\varepsilon}$ is the boundary of our porous medium $\Omega \backslash \overline{\Omega^{\varepsilon}}$. Moreover, the fluid is assumed to be homogeneous and isotropic, with a constant diffusion coefficient $D>0$. In (1.1)-(1.5), $v^{\varepsilon}$ can be interpreted as being the concentration of the solute in the fluid region, $w^{\varepsilon}$ as the concentration of the solute on the surface of the skeleton $\Omega \backslash \overline{\Omega^{\varepsilon}}, v_{1}$ as the initial concentration of the solute and $w_{1}$ as the initial concentration of the reactants on the surface $S^{\varepsilon}$ of the skeleton; $a$ and $\gamma$ are called the reaction factor and the adsorption factor, respectively.

The semilinear boundary condition on $S^{\varepsilon}$ (see (1.2), (1.4)) in problem (1.1)-(1.4) describes the interchanges of chemical flows across the boundary $S^{\varepsilon}$. They are governed by a general non-linear balance law involving two main ingredients, namely an adsorption factor $\gamma$ (which, in a first step, we will assume to be constant) and the so-called adsorption rate which concentrates the non-linear behavior of this equilibrium; it is represented by $g$ in (1.2)-(1.4), which is assumed to be given. Two model situations will be considered: the case in which $g$ is a monotone smooth function satisfying the condition $g(0)=0$ and the case of a maximal monotone graph with $g(0)=0$, i.e. the case in which $g$ is the subdifferential of a convex lower semicontinuous function $G$. These two general situations are well illustrated by the following important practical examples (see [13] and [18]):

$$
\text { a) } g(v)=\frac{\alpha v}{1+\beta v}, \quad \alpha, \beta>0 \quad \text { (Langmuir kinetics) }
$$

and

$$
\text { b) } g(v)=|v|^{p-1} v, \quad 0<p<1 \quad \text { (Freundlich kinetics). }
$$

The exponent $p$ in example $b$ ) is called the order of the reaction. In some applications the limit case $(p=0)$ is of great relevance (see Remark 3.5). It is worth remarking that if $v^{\varepsilon} \geq 0$ in $\overline{\Omega^{\varepsilon}}$ and $v^{\varepsilon}>0$ in $\Omega^{\varepsilon}$, then the function $g$ in example $a$ ) is indeed a particular example of our first model situation ( $g$ is a monotone smooth function satisfying the condition $g(0)=0$ ). Also, let us note that, instead of (1.4), we could consider a more general boundary condition, given in terms of

$$
f^{\varepsilon}(t, x)=\gamma_{1} g\left(v^{\varepsilon}(t, x)\right)-\gamma_{2} w^{\varepsilon}(t, x),
$$

where $\gamma_{1}>0$ is called adsorption factor and $\gamma_{2}>0$ is called desorption factor (see [19]).

The existence and uniqueness of a weak solution of the system (1.1)-(1.4) can be settled by using the classical theory of semilinear monotone problems (see, for instance, [7] and [20]). As a result, we know that there exists a unique weak solution $u^{\varepsilon}=\left(v^{\varepsilon}, w^{\varepsilon}\right)$.

From a geometrical point of view, we shall just consider periodic structures obtained by removing periodically from $\Omega$, with period $\varepsilon Y$ (where $Y$ is a given hyper-rectangle in $\mathbb{R}^{n}$ ), an elementary hole $F$ which has been appropriated rescaled and which is strictly included in $Y$, i.e. $\bar{F} \subset Y$.

As usual in homogenization, we shall be interested in obtaining a suitable description of the asymptotic behavior, as $\varepsilon$ tends to zero, of the solution $u^{\varepsilon}$ in such domains. We will wonder, for example, whether the solution $u^{\varepsilon}$ converges to a limit $u$ as $\varepsilon \rightarrow 0$. And if this limit exists, can it be characterized? 
If we denote by $\star$ the convolution with respect to time and if

$$
r(\rho)=e^{-(a+\gamma) \rho},
$$

then we prove that the solution $v^{\varepsilon}$, properly extended to the whole of $\Omega$, converges to the unique solution $v$ (effective behavior) of the following problem:

$$
(V)\left\{\begin{array}{l}
\frac{\partial v}{\partial t}(t, x)-D \sum_{i, j=1}^{n} q_{i j} \frac{\partial^{2} v}{\partial x_{i} \partial x_{j}}(t, x)+F_{0}(t, x)=h(t, x), \quad t>0, x \in \Omega, \\
v(t, x)=0, \quad t>0, x \in \partial \Omega \\
v(t, x)=v_{1}(x), \quad t=0, x \in \Omega
\end{array}\right.
$$

with

$$
F_{0}(t, x)=\frac{|\partial F|}{|Y \backslash \bar{F}|} \gamma\left[g(v(t, x))-w_{1}(x) e^{-(a+\gamma) t}-\gamma r(\cdot) \star g(v(\cdot, x))(t)\right] .
$$

In (1.6), $Q=\left(\left(q_{i j}\right)\right)$ is the classical homogenized matrix, whose entries are defined as follows:

$$
q_{i j}=\delta_{i j}+\frac{1}{|Y \backslash \bar{F}|} \int_{Y \backslash \bar{F}} \frac{\partial \chi_{j}}{\partial y_{i}} d y
$$

in terms of the functions $\chi_{i}, i=1, \ldots, n$, solutions of the so-called cell problems

$$
\left\{\begin{array}{l}
-\Delta \chi_{i}=0 \text { in } Y \backslash \bar{F} \\
\frac{\partial\left(\chi_{i}+y_{i}\right)}{\partial \nu}=0 \text { on } \partial F \\
\chi_{i} Y-\text { periodic. }
\end{array}\right.
$$

Moreover, let us notice that the limit problem for the surface concentration $w$ is

$$
\left\{\begin{array}{l}
\frac{\partial w}{\partial t}(t, x)+(a+\gamma) w(t, x)=\gamma g(v(t, x)), \quad t>0, x \in \Omega \\
w(t, x)=w_{1}(x), \quad t=0, x \in \Omega
\end{array}\right.
$$

and obviously $w$ can be written as

$$
w(t, x)=w_{1}(x) e^{-(a+\gamma) t}+\gamma r(\cdot) \star g(v(\cdot, x))(t) .
$$

The macroscopic problem (1.6) arises from the homogenization of a boundary-value problem in a periodically perforated domain and the zero-order term occurring in (1.6) has its origin in this particular structure of the model. The influence of the adsorption and chemical reactions taking place on the boundaries of the perforations is reflected by the appearance of this zero-order extra-term.

In problem (1.1)-(1.4), the rate of chemical reactions on $S^{\varepsilon}$, namely $a$, and the adsorption coefficient $\gamma$ were assumed to be constant. From a practical point of view, a more realistic model would be to assume that the surface $\partial F$ is chemically and physically heterogeneous, which means that $a$ and $\gamma$ are rapidly oscillating functions. Moreover, one can consider a more general model, including the diffusion of the chemical species on the surface $S^{\varepsilon}$. In fact, the chemical substances can creep on the surface and this effect is similar to a surface-like diffusion. From a mathematical point of view, we can model this phenomenon by introducing a diffusion term in the law governing 
the evolution of the surface concentration $w^{\varepsilon}$ (see (1.3)). This new term is nothing but the LaplaceBeltrami operator properly rescaled. Taking into account all these generalizations, we are lead to system (4.5)-(4.8) (see Chapter 4). The limit problem in this case is almost the same as before, except that it involves the solution of a reaction-diffusion system with respect to an additional microvariable. Also, notice that the local behavior is no longer governed by an ordinary differential equation, but by a partial differential one (see (4.12)).

The structure of our paper is as follows: first, let us mention that we shall just focus on the case $n \geq 3$, which will be treated explicitly. The case $n=2$ is much more simpler and we shall omit to treat it. In Chapter 2 we consider the simpler case of chemical flows just involving homogeneous adsorption and chemical reactions. We start by stating some preliminary notation and assumptions, we give a rigorous setting of the problem and we formulate the main convergence result, the proof of which is given in Chapter 3. Chapter 4 is devoted to treat a more general model, namely the case where the surface of the grains is heterogeneous and we have also diffusion thereon.

Finally, notice that throughout the paper, by $C$ we shall denote a generic fixed strictly positive constant, whose value can change from line to line.

\section{Preliminaries and main result}

In this chapter, we will be concerned with some preliminary notation and assumptions, as well as with the rigorous setting of our main model, involving the simpler case of adsorption and chemical reactions. As already mentioned in the Introduction, our main result provides the effective behavior of the reactive flows (see Section 2.3).

\subsection{Notation and assumptions}

\subsubsection{The geometry of the problem}

Let $\Omega$ be a smooth bounded connected open subset of $\mathbb{R}^{n}(n \geq 3)$ and let $Y=\left[0, l_{1}\left[\times \ldots\left[0, l_{n}[\right.\right.\right.$ be the representative cell in $\mathbb{R}^{n}$. Denote by $F$ an open subset of $Y$ with smooth boundary $\partial F$ such that $\bar{F} \subset Y$. We shall refer to $F$ as being the elementary hole. Also, let $[0, T]$, with $T>0$, be the time interval of interest.

Let $\varepsilon$ be a real parameter taking values in a sequence of positive numbers converging to zero. For each $\varepsilon$ and for any integer vector $k \in \mathbb{Z}^{n}$, set $T_{k}^{\varepsilon}$ the translated image of $\varepsilon F$ by the vector $k l=\left(k_{1} l_{1}, \ldots, k_{n} l_{n}\right)$ :

$$
F_{k}^{\varepsilon}=\varepsilon(k l+F) .
$$

The set $F_{k}^{\varepsilon}$ represents the holes in $\mathbb{R}^{n}$. Also, let us denote by $F^{\varepsilon}$ the set of all the holes contained in $\Omega$, i.e.

$$
F^{\varepsilon}=\bigcup\left\{F_{k}^{\varepsilon} \mid \overline{F_{k}^{\varepsilon}} \subset \Omega, k \in \mathbb{Z}^{n}\right\}
$$

Set

$$
\Omega^{\varepsilon}=\Omega \backslash \overline{F^{\varepsilon}} .
$$

Hence, $\Omega^{\varepsilon}$ is a periodically perforated domain with holes of size of the same order as the period. Remark that the holes do not intersect the boundary $\partial \Omega$.

Let

$$
S^{\varepsilon}=\cup\left\{\partial F_{k}^{\varepsilon} \mid \overline{F_{k}^{\varepsilon}} \subset \Omega, k \in \mathbb{Z}^{n}\right\} .
$$

So

$$
\partial \Omega^{\varepsilon}=\partial \Omega \cup S^{\varepsilon} .
$$


We shall also use the following notations:

$$
\begin{aligned}
& |\omega|=\text { the Lebesgue measure of any measurable subset } \omega \text { of } \mathbb{R}^{n}, \\
& \qquad \chi_{\omega}=\text { the characteristic function of the set } \omega, \\
& \qquad Y^{*}=Y \backslash \bar{F},
\end{aligned}
$$

and

$$
\theta=\frac{\left|Y^{*}\right|}{|Y|}
$$

\subsubsection{Function spaces and norms}

In the sequel, we shall use the following notations:

$$
H=L^{2}(\Omega)
$$

with the classical scalar product and norm:

$$
\begin{gathered}
(u, v)_{\Omega}=\int_{\Omega} u(x) v(x) d x, \quad\|u\|_{\Omega}^{2}=(u, u)_{\Omega}, \\
\mathcal{H}=L^{2}(0, T ; H)
\end{gathered}
$$

with

$$
\begin{gathered}
(u, v)_{\Omega, T}=\int_{0}^{T}(u(t), v(t))_{\Omega} d t, \text { where } u(t)=u(t, \cdot), v(t)=v(t, \cdot),\|u\|_{\Omega, T}^{2}=(u, u)_{\Omega, T} \\
V=H^{1}(\Omega)
\end{gathered}
$$

with

$$
\begin{gathered}
(u, v)_{V}=(u, v)_{\Omega}+(\nabla u, \nabla v)_{\Omega} \\
\mathcal{V}=L^{2}(0, T ; V)
\end{gathered}
$$

with

$$
\begin{gathered}
(u, v)_{\mathcal{V}}=\int_{0}^{T}(u(t), v(t))_{V} d t \\
\mathcal{W}=\left\{v \in \mathcal{V} \mid \frac{d v}{d t} \in \mathcal{V}^{\prime}\right\} \quad \text { where } \mathcal{V}^{\prime} \text { is the dual space of } \mathcal{V}, \\
\mathcal{V}_{0}=\{v \in \mathcal{V} \mid v=0 \text { on } \partial \Omega \text { a.e. on }(0, T)\}, \\
\mathcal{W}_{0}=\mathcal{V}_{0} \bigcap \mathcal{W} .
\end{gathered}
$$

Similarly, we define the spaces $V\left(\Omega^{\varepsilon}\right), \mathcal{V}\left(\Omega^{\varepsilon}\right), V\left(S^{\varepsilon}\right)$ and $\mathcal{V}\left(S^{\varepsilon}\right)$. For the latter we write

$$
\langle u, v\rangle_{S^{\varepsilon}}=\int_{S^{\varepsilon}} g^{\varepsilon} u v d \sigma,
$$


where $g^{\varepsilon}$ is the metric tensor on $S^{\varepsilon}$; the rule of partial integration on $S^{\varepsilon}$ applies and, if we denote the gradient on $S^{\varepsilon}$ by $\nabla^{\varepsilon}$ and the Laplace-Beltrami operator on $S^{\varepsilon}$ by $\Delta^{\varepsilon}$, we have

$$
-\left(\Delta^{\varepsilon} u, v\right)_{S^{\varepsilon}}=\left\langle\nabla^{\varepsilon} u, \nabla^{\varepsilon} v\right\rangle_{S^{\varepsilon}} .
$$

Also, for the space of test functions we use the notation

$$
\left.\mathcal{D}=C_{0}^{\infty}((0, T) \times \Omega)\right) .
$$

Moreover, for an arbitrary function $\psi \in L^{2}\left(\Omega^{\varepsilon}\right)$, we shall denote by $\widetilde{\psi}$ its extension by zero inside the holes:

$$
\widetilde{\psi}=\left\{\begin{array}{lll}
\psi & \text { in } & \Omega^{\varepsilon} \\
0 & \text { in } & \Omega \backslash \overline{\Omega^{\varepsilon}}
\end{array}\right.
$$

and, also, for any open subset $D \subset \mathbb{R}^{n}$ and for any function $g \in L^{1}(D)$, we set

$$
\mathcal{M}_{D}(g)=\frac{1}{|D|} \int_{D} g d x
$$

\subsection{Setting of the problem}

As already mentioned, we are interested in studying the behavior of the solution $u^{\varepsilon}=\left(v^{\varepsilon}, w^{\varepsilon}\right)$, in such a perforated domain, of the following problem:

$$
\begin{gathered}
\left(V^{\varepsilon}\right)\left\{\begin{array}{l}
\frac{\partial v^{\varepsilon}}{\partial t}(t, x)-D \Delta v^{\varepsilon}(t, x)=h(t, x), \quad x \in \Omega^{\varepsilon}, t>0 \\
v^{\varepsilon}(t, x)=0, \quad x \in \partial \Omega, t>0 \\
v^{\varepsilon}(t, x)=v_{1}(x), \quad x \in \Omega^{\varepsilon}, t=0
\end{array}\right. \\
\quad-D \frac{\partial v^{\varepsilon}}{\partial \nu}(t, x)=\varepsilon f^{\varepsilon}(t, x), \quad x \in S^{\varepsilon}, t>0
\end{gathered}
$$

and

$$
\left(W^{\varepsilon}\right)\left\{\begin{aligned}
& \frac{\partial w^{\varepsilon}}{\partial t}(t, x)+a w^{\varepsilon}(t, x)=f^{\varepsilon}(t, x), \quad x \in S^{\varepsilon}, t>0 \\
& w^{\varepsilon}(t, x)=w_{1}(x), \quad x \in S^{\varepsilon}, t=0
\end{aligned}\right.
$$

where

$$
f^{\varepsilon}(t, x)=\gamma\left(g\left(v^{\varepsilon}(t, x)\right)-w^{\varepsilon}(t, x)\right) .
$$

Here, $\nu$ is the exterior unit normal to $\Omega^{\varepsilon}, a, \gamma>0, h \in \mathcal{H}, v_{1}, w_{1} \in H_{0}^{1}(\Omega)$ and $S^{\varepsilon}$ is the boundary of our porous medium $\Omega \backslash \overline{\Omega^{\varepsilon}}$. Moreover, the fluid is assumed to be homogeneous and isotropic, with a constant diffusion coefficient $D>0$.

The function $g$ in (2.6) is assumed to be given. Two model situations will be considered; the case in which $g$ is a monotone smooth function satisfying the condition $g(0)=0$ and the case of a maximal monotone graph with $g(0)=0$, i.e. the case in which $g$ is the subdifferential of a convex lower semicontinuous function $G$. These two general situations are well illustrated by the following important practical examples:

$$
\text { a) } g(v)=\frac{\alpha v}{1+\beta v}, \quad \alpha, \beta>0 \quad \text { (Langmuir kinetics) }
$$

and

$$
\text { b) } g(v)=|v|^{p-1} v, \quad 0<p<1 \quad \text { (Freundlich kinetics). }
$$


The existence and uniqueness of a weak solution of the system (2.3)-(2.6) can be settled by using the classical theory of semilinear monotone problems (see, for instance, [7] and [20]).

We shall first consider the case in which $g$ is a continuously differentiable function, monotonously non-decreasing and such that $g(v)=0$ iff $v=0$. Also, we shall suppose that there exist a positive constant $C$ and an exponent $q$, with $0 \leq q<n /(n-2)$, such that

$$
\left|\frac{\partial g}{\partial v}\right| \leq C\left(1+|v|^{q}\right) \text {. }
$$

As we will see later on this hypothesis concerning the smoothness of the nonlinearity $g$ is not fundamental and can be easily overcame by using a regularization technique, for example Yosida approximation (see Section 3.4).

The weak formulation of problem (2.3)-(2.6) is:

$$
\left\{\begin{array}{l}
\text { Find } v^{\varepsilon} \in \mathcal{W}_{0}\left(\Omega^{\varepsilon}\right), v^{\varepsilon}(0)=\left.v_{1}\right|_{\Omega^{\varepsilon}} \text { such that } \\
-\left(v^{\varepsilon}, \frac{d \varphi}{d t}\right)_{\Omega^{\varepsilon}, T}+\varepsilon\left(f^{\varepsilon}, \varphi\right)_{\Omega^{\varepsilon}, T}=-D\left(\nabla v^{\varepsilon}, \nabla \varphi\right)_{\Omega^{\varepsilon}, T}+(h, \varphi)_{\Omega^{\varepsilon}, T}, \quad \forall \varphi \in \mathcal{W}_{0}\left(\Omega^{\varepsilon}\right)
\end{array}\right.
$$

and

$$
\left\{\begin{array}{l}
\text { Find } w^{\varepsilon} \in \mathcal{W}\left(S^{\varepsilon}\right), w^{\varepsilon}(0)=\left.w_{1}\right|_{S^{\varepsilon}} \text { such that } \\
-\left(w^{\varepsilon}, \frac{d \varphi}{d t}\right)_{S^{\varepsilon}, T}+a\left(w^{\varepsilon}, \varphi\right)_{S^{\varepsilon}, T}=\left(f^{\varepsilon}, \varphi\right)_{S^{\varepsilon}, T}, \quad \forall \varphi \in \mathcal{W}\left(S^{\varepsilon}\right) .
\end{array}\right.
$$

By classical existence results (see [7] and [20]), there exists a unique weak solution $u^{\varepsilon}=\left(v^{\varepsilon}, w^{\varepsilon}\right)$ of the system (2.8)-(2.9).

Remark 2.1 Let us notice that the solution of (2.5) can be written as

$$
w^{\varepsilon}(t, x)=w_{1}(x) e^{-(a+\gamma) t}+\gamma \int_{0}^{t} e^{-(a+\gamma)(t-s)} g\left(v^{\varepsilon}(s, x)\right) d s
$$

or, if we denote by $\star$ the convolution with respect to time, as

$$
w^{\varepsilon}(\cdot, x)=w_{1}(x) e^{-(a+\gamma) t}+\gamma r(\cdot) \star g\left(v^{\varepsilon}(\cdot, x)\right),
$$

where

$$
r(\rho)=e^{-(a+\gamma) \rho} .
$$

The solution $v^{\varepsilon}$ of problem $\left(V^{\varepsilon}\right)$ being defined only on $\Omega^{\varepsilon}$, we need to extend it to the whole of $\Omega$ to be able to state the convergence result. In order to do that, let us recall the following well-known extension result (see [10] and [12]):

Lemma 2.2 There exists a linear continuous extension operator $P^{\varepsilon} \in \mathcal{L}\left(L^{2}\left(\Omega^{\varepsilon}\right) ; L^{2}(\Omega)\right) \cap$ $\cap \mathcal{L}\left(V^{\varepsilon} ; H_{0}^{1}(\Omega)\right)$ and a positive constant $C$, independent of $\varepsilon$, such that

$$
\left\|P^{\varepsilon} v\right\|_{L^{2}(\Omega)} \leq C\|v\|_{L^{2}\left(\Omega^{\varepsilon}\right)}
$$

and

for any $v \in V^{\varepsilon}$, where

$$
\left\|\nabla P^{\varepsilon} v\right\|_{L^{2}(\Omega)} \leq C\|\nabla v\|_{L^{2}\left(\Omega^{\varepsilon}\right)}
$$

$$
V^{\varepsilon}=\left\{v \in H^{1}\left(\Omega^{\varepsilon}\right) \mid v=0 \text { on } \partial \Omega\right\},
$$

with

$$
\|v\|_{V^{\varepsilon}}=\|\nabla v\|_{L^{2}\left(\Omega^{\varepsilon}\right)} \cdot
$$


An immediate consequence of the previous lemma is the following Poincaré's inequality in $V^{\varepsilon}$ :

Lemma 2.3 There exists a positive constant $C$, independent of $\varepsilon$, such that

$$
\|v\|_{L^{2}\left(\Omega^{\varepsilon}\right)} \leq C\|\nabla v\|_{L^{2}\left(\Omega^{\varepsilon}\right)},
$$

for any $v \in V^{\varepsilon}$.

We also recall the following well-known result (see [11]):

Lemma 2.4 There exists a positive constant $C$, independent of $\varepsilon$, such that

$$
\|v\|_{L^{2}\left(S^{\varepsilon}\right)}^{2} \leq C\left(\varepsilon^{-1}\|v\|_{L^{2}\left(\Omega^{\varepsilon}\right)}^{2}+\varepsilon\|\nabla v\|_{L^{2}\left(\Omega^{\varepsilon}\right)}^{2}\right),
$$

for any $v \in V^{\varepsilon}$.

\subsection{The main result}

The main result of this paper is the following one:

Theorem 2.5 One can construct an extension $P^{\varepsilon} v^{\varepsilon}$ of the solution $v^{\varepsilon}$ of the problem $\left(V^{\varepsilon}\right)$ such that

$$
P^{\varepsilon} v^{\varepsilon} \rightarrow v \quad \text { weakly in } \mathcal{V}
$$

where $v$ is the unique solution of the following limit problem:

$$
\left\{\begin{array}{l}
\frac{\partial v}{\partial t}(t, x)+F_{0}(t, x)-D \sum_{i, j=1}^{n} q_{i j} \frac{\partial^{2} v}{\partial x_{i} \partial x_{j}}(t, x)=h(t, x), \quad t>0, x \in \Omega, \\
v(t, x)=0, \quad t>0, x \in \partial \Omega \\
v(t, x)=v_{1}(x), \quad t=0, x \in \Omega
\end{array}\right.
$$

with

$$
F_{0}(t, x)=\frac{|\partial F|}{\left|Y^{\star}\right|} \gamma\left[g(v(t, x))-w_{1}(x) e^{-(a+\gamma) t}-\gamma r(\cdot) \star g(v(\cdot, x))(t)\right] .
$$

In (2.13), $Q=\left(\left(q_{i j}\right)\right)$ is the classical homogenized matrix, whose entries are defined as follows:

$$
q_{i j}=\delta_{i j}+\frac{1}{\left|Y^{*}\right|} \int_{Y^{*}} \frac{\partial \chi_{j}}{\partial y_{i}} d y
$$

in terms of the functions $\chi_{i}, i=1, \ldots, n$, solutions of the so-called cell problems

$$
\left\{\begin{array}{l}
-\Delta \chi_{i}=0 \text { in } Y^{*} \\
\frac{\partial\left(\chi_{i}+y_{i}\right)}{\partial \nu}=0 \text { on } \partial F \\
\chi_{i} Y-\text { periodic. }
\end{array}\right.
$$

The constant matrix $Q$ is symmetric and positive-definite. Moreover, the limit problem for the surface concentration is:

$$
\left\{\begin{array}{l}
\frac{\partial w}{\partial t}(t, x)+(a+\gamma) w(t, x)=\gamma g(v(t, x)), \quad t>0, x \in \Omega \\
w(t, x)=w_{1}(x), \quad t=0, x \in \Omega
\end{array}\right.
$$

and obviously, $w$ can be written as

$$
w(t, x)=w_{1}(x) e^{-(a+\gamma) t}+\gamma r(t) \star g(v(t, x))
$$


Remark 2.6 The weak formulation of problem (2.13) is:

$$
\left\{\begin{array}{l}
\text { Find } v \in \mathcal{W}_{0}(\Omega), v(0)=v_{1} \text { such that } \\
-\left(v, \frac{d \varphi}{d t}\right)_{\Omega, T}+\left(F_{0}, \varphi\right)_{\Omega, T}=-D(Q \nabla v, \nabla \varphi)_{\Omega, T}+(h, \varphi)_{\Omega, T} \\
\forall \varphi \in \mathcal{W}_{0}(\Omega)
\end{array}\right.
$$

\section{Proof of the main result}

\subsection{Uniqueness of the limit problem (2.18)}

The proof of Theorem 2.5 consists of several different steps, the first one being to prove uniqueness of the limit problem (2.18). This is state in the following

Proposition 3.1 There is at most one solution of the weak problem (2.18). .

Proof. Let us suppose that there exist two solutions $v^{1}, v^{2} \in \mathcal{W}_{0}(\Omega)$ of the weak problem (2.18) and let $v=v^{1}-v^{2}$. Denote

$$
\alpha=\gamma \frac{|\partial F|}{\left|Y^{\star}\right|} .
$$

Obviously $v \in \mathcal{W}_{0}(\Omega), v(0)=0$ and $v$ satisfies

$$
\left(\frac{\partial v}{\partial t}, \varphi\right)_{\Omega}+\alpha\left(g\left(v^{1}\right)-g\left(v^{2}\right), \varphi\right)_{\Omega}-\alpha \gamma\left(r \star\left(g\left(v^{1}\right)-g\left(v^{2}\right)\right), \varphi\right)_{\Omega}=-D(Q \nabla v, \nabla \varphi)_{\Omega}
$$

a.e. on $(0, T)$ and for all $\varphi \in \mathcal{V}_{0}(\Omega)$. We intent to prove that $v=0$. Taking $v$ as a test function in (3.1), we get

$$
\frac{1}{2} \frac{d}{d t}\|v(t)\|_{\Omega}^{2}+\alpha\left(g\left(v^{1}\right)-g\left(v^{2}\right), v\right)_{\Omega}-\alpha \gamma\left(r \star\left(g\left(v^{1}\right)-g\left(v^{2}\right)\right), v\right)_{\Omega}=-D(Q \nabla v, \nabla v)_{\Omega} .
$$

Therefore

$$
\frac{1}{2} \frac{d}{d t}\|v(t)\|_{\Omega}^{2}+\alpha\left(g\left(v^{1}\right)-g\left(v^{2}\right), v^{1}-v^{2}\right)_{\Omega} \leq \alpha \gamma\left(r \star\left(g\left(v^{1}\right)-g\left(v^{2}\right)\right), v^{1}-v^{2}\right)_{\Omega} .
$$

Integrating from 0 to $t$ and taking into account the assumptions we have made for $g$, we get

$$
\frac{1}{2}\|v(t)\|_{\Omega}^{2} \leq \alpha \gamma \int_{0}^{t}\left[\int_{\Omega}\left(r \star\left(g\left(v^{1}\right)-g\left(v^{2}\right)\right)\right)(s)\left(v^{1}-v^{2}\right)(s) d x\right] d s .
$$

Let us evaluate now the right-hand side of (3.2). We have

$$
\begin{gathered}
\left|\int_{\Omega}\left(\int_{0}^{s} r(s-\sigma)\left(g\left(v^{1}(\sigma, x)\right)-g\left(v^{2}(\sigma, x)\right)\right) d \sigma\right)\left(v^{1}-v^{2}\right)(s, x) d x\right| \leq \\
\leq C \int_{0}^{s}\left\|\left(g\left(v^{1}\right)-g\left(v^{2}\right)\right)(\sigma)\right\|_{\Omega}\|v(s)\| d \sigma \leq \\
\leq C\left\{\frac{1}{2} \int_{0}^{s}\left\|\left(g\left(v^{1}\right)-g\left(v^{2}\right)\right)(\sigma)\right\|_{\Omega}^{2}+\frac{s}{2}\|v(s)\|_{\Omega}^{2}\right\} .
\end{gathered}
$$


Integrating over $s$ between 0 and $t$, we get

$$
\begin{gathered}
\int_{0}^{t}\left|\int_{\Omega}\left(\int_{0}^{s} r(s-\sigma)\left(g\left(v^{1}(\sigma, x)\right)-g\left(v^{2}(\sigma, x)\right)\right) d \sigma\right)\left(v^{1}-v^{2}\right)(s, x) d x\right| d s \leq \\
\leq C\left\{\int_{0}^{t} \int_{0}^{s}\left\|\left(g\left(v^{1}\right)-g\left(v^{2}\right)\right)(\sigma)\right\|_{\Omega}^{2} d \sigma d s+s\|v(s)\|_{\Omega}^{2} d s\right\}= \\
=C\left\{\int_{0}^{t} \int_{\sigma}^{t} d s\left\|\left(g\left(v^{1}\right)-g\left(v^{2}\right)\right)(\sigma)\right\|_{\Omega}^{2} d \sigma+\int_{0}^{t} s\|v(s)\|_{\Omega}^{2} d s\right\} \leq \\
\leq C\left\{\int_{0}^{t} \int_{\sigma}^{t} d s\left\|\left(v^{1}-v^{2}\right)(\sigma)\right\|_{\Omega}^{2} d \sigma+\int_{0}^{t} s\|v(s)\|_{\Omega}^{2} d s\right\} \leq C t \int_{0}^{t}\|v(s)\|_{\Omega}^{2} d s, \quad \forall t \leq T .
\end{gathered}
$$

Finally, from (3.2) we obtain

$$
\|v(t)\|_{\Omega}^{2} \leq C \int_{0}^{t}\|v(s)\|_{\Omega}^{2} d s
$$

and hence, using Gronwall's inequality, we get $v \equiv 0$.

\subsection{A priori estimates}

The second step of the proof of Theorem 2.5 consists in describing the effective behavior of $v^{\varepsilon}$ and $w^{\varepsilon}$, as $\varepsilon \rightarrow 0$. To this end, some a priori estimates on these solutions are required.

Proposition 3.2 Let $v^{\varepsilon}$ and $w^{\varepsilon}$ be the solutions of the problem (2.3)-(2.6). There exists a positive constant $C$, independent of $\varepsilon$, such that

and

$$
\begin{gathered}
\left\|w^{\varepsilon}(t)\right\|_{S^{\varepsilon}}^{2} \leq\left(\left\|w^{\varepsilon}(0)\right\|_{S^{\varepsilon}}^{2}+\frac{\gamma}{\delta}\left\|g\left(v^{\varepsilon}\right)\right\|_{S^{\varepsilon}, t}^{2}\right) e^{\gamma \delta t}, \quad \forall t \geq 0, \forall \delta>0 \\
\left\|\frac{\partial w^{\varepsilon}}{\partial t}\right\|_{S^{\varepsilon}, t}^{2} \leq C\left(\left\|w^{\varepsilon}(0)\right\|_{S^{\varepsilon}}^{2}+\left\|g\left(v^{\varepsilon}\right)\right\|_{S^{\varepsilon}, t}^{2}\right), \quad \forall t \geq 0 \\
\left\|v^{\varepsilon}(t)\right\|_{\Omega^{\varepsilon}}^{2} \leq C,\left\|\nabla v^{\varepsilon}(t)\right\|_{\Omega^{\varepsilon}, t}^{2} \leq C
\end{gathered}
$$

$$
\left\|\frac{\partial v^{\varepsilon}}{\partial t}(t)\right\|_{\Omega^{\varepsilon}}^{2} \leq C
$$

Proof. From (2.5) we obtain

$$
\int_{S^{\varepsilon}} \frac{\partial w^{\varepsilon}}{\partial t} w^{\varepsilon} d \sigma+\int_{S^{\varepsilon}}(a+\gamma)\left(w^{\varepsilon}\right)^{2} d \sigma=\int_{S^{\varepsilon}} \gamma g\left(v^{\varepsilon}\right) w^{\varepsilon} d \sigma .
$$

Therefore

$$
\frac{1}{2} \frac{d}{d t}\left\|w^{\varepsilon}\right\|_{S^{\varepsilon}}^{2}+(a+\gamma)\left\|w^{\varepsilon}\right\|_{S^{\varepsilon}}^{2} \leq \gamma\left\|g\left(v^{\varepsilon}\right)\right\|_{S^{\varepsilon}}\left\|w^{\varepsilon}\right\|_{S^{\varepsilon}} \leq
$$




$$
\leq \gamma\left(\frac{\left\|g\left(v^{\varepsilon}\right)\right\|_{S^{\varepsilon}}^{2}}{2 \delta}+\frac{\delta}{2}\left\|w^{\varepsilon}\right\|_{S^{\varepsilon}}^{2}\right), \quad \forall \delta>0 .
$$

Integrating with respect to $t$ and using Gronwall's inequality, we get

$$
\left\|w^{\varepsilon}(t)\right\|_{S^{\varepsilon}}^{2} \leq\left(\left\|w^{\varepsilon}(0)\right\|_{S^{\varepsilon}}^{2}+\frac{\gamma}{\delta}\left\|g\left(v^{\varepsilon}\right)\right\|_{S^{\varepsilon}, t}^{2}\right) e^{\gamma \delta t}, \quad \forall t \geq 0, \forall \delta>0 .
$$

In a similar manner we can obtain

$$
\left\|\frac{\partial w^{\varepsilon}}{\partial t}\right\|_{S^{\varepsilon}, t}^{2} \leq C\left(\left\|w^{\varepsilon}(0)\right\|_{S^{\varepsilon}}^{2}+\left\|g\left(v^{\varepsilon}\right)\right\|_{S^{\varepsilon}, t}^{2}\right), \quad \forall t \geq 0 .
$$

Let us now prove (3.5). Multiplying (2.3) by $v^{\varepsilon}$, using (2.4)-(2.6) and integrating over $\Omega^{\varepsilon}$, we have

$$
\int_{\Omega^{\varepsilon}} \frac{\partial v^{\varepsilon}}{\partial t} v^{\varepsilon} d x+D \int_{\Omega^{\varepsilon}} \nabla v^{\varepsilon} \nabla v^{\varepsilon} d x+\varepsilon \int_{S^{\varepsilon}} \gamma\left(g\left(v^{\varepsilon}\right)-w^{\varepsilon}\right) v^{\varepsilon} d \sigma=\int_{\Omega^{\varepsilon}} h v^{\varepsilon} d x .
$$

Therefore

$$
\begin{gathered}
\frac{1}{2} \frac{d}{d t}\left\|v^{\varepsilon}\right\|_{\Omega^{\varepsilon}}^{2}+D\left\|\nabla v^{\varepsilon}\right\|_{\Omega^{\varepsilon}}^{2}+\varepsilon \gamma \int_{S^{\varepsilon}} g\left(v^{\varepsilon}\right) v^{\varepsilon} d \sigma=\varepsilon \gamma \int_{S^{\varepsilon}} w^{\varepsilon} v^{\varepsilon} d \sigma+\int_{\Omega^{\varepsilon}} h v^{\varepsilon} d x \leq \\
\leq \varepsilon \gamma\left\|w^{\varepsilon}\right\|_{S^{\varepsilon}}\left\|v^{\varepsilon}\right\|_{S^{\varepsilon}}+C\left\|v^{\varepsilon}\right\|_{\Omega^{\varepsilon}} \leq \\
\leq \varepsilon \gamma\left\|w^{\varepsilon}\right\|_{S^{\varepsilon}}\left\|v^{\varepsilon}\right\|_{S^{\varepsilon}}+\frac{D}{2}\left\|\nabla v^{\varepsilon}\right\|_{\Omega^{\varepsilon}}^{2}+C .
\end{gathered}
$$

Hence

$$
\frac{1}{2} \frac{d}{d t}\left\|v^{\varepsilon}\right\|_{\Omega^{\varepsilon}}^{2}+\frac{D}{2}\left\|\nabla v^{\varepsilon}\right\|_{\Omega^{\varepsilon}}^{2}+\varepsilon \gamma \int_{S^{\varepsilon}} g\left(v^{\varepsilon}\right) v^{\varepsilon} d \sigma \leq \varepsilon \gamma\left\|w^{\varepsilon}\right\|_{S^{\varepsilon}}\left\|v^{\varepsilon}\right\|_{S^{\varepsilon}}+C .
$$

Using Young's inequality and (3.3), we get

$$
\begin{gathered}
\frac{1}{2} \frac{d}{d t}\left\|v^{\varepsilon}\right\|_{\Omega^{\varepsilon}}^{2}+\frac{D}{2}\left\|\nabla v^{\varepsilon}\right\|_{\Omega^{\varepsilon}}^{2}+\varepsilon \gamma \int_{S^{\varepsilon}} g\left(v^{\varepsilon}\right) v^{\varepsilon} d \sigma \leq C_{1} \varepsilon\left\|w^{\varepsilon}\right\|_{S^{\varepsilon}}^{2}+C_{2} \varepsilon\left\|v^{\varepsilon}\right\|_{S^{\varepsilon}}^{2}+C \leq \\
\leq C_{3} \varepsilon\left\|w^{\varepsilon}(0)\right\|_{S^{\varepsilon}}^{2}+C_{4} \varepsilon\left\|g\left(v^{\varepsilon}\right)\right\|_{S^{\varepsilon}}^{2}+C_{2} \varepsilon\left\|v^{\varepsilon}\right\|_{S^{\varepsilon}}^{2}+C .
\end{gathered}
$$

Using Lemma 2.4 and our hypotheses for $g$ and $w_{1}$, we easily get

$$
\frac{1}{2} \frac{d}{d t}\left\|v^{\varepsilon}\right\|_{\Omega^{\varepsilon}}^{2}+\frac{D}{2}\left\|\nabla v^{\varepsilon}\right\|_{\Omega^{\varepsilon}}^{2}+\varepsilon \gamma \int_{S^{\varepsilon}} g\left(v^{\varepsilon}\right) v^{\varepsilon} d \sigma \leq C_{5}\left\|v^{\varepsilon}\right\|_{\Omega^{\varepsilon}}^{2}+C_{0} \varepsilon^{2}\left\|\nabla v^{\varepsilon}\right\|_{\Omega^{\varepsilon}}^{2}+K .
$$

Then

$$
\frac{1}{2} \frac{d}{d t}\left\|v^{\varepsilon}\right\|_{\Omega^{\varepsilon}}^{2}+\left(\frac{D}{2}-C_{0} \varepsilon^{2}\right)\left\|\nabla v^{\varepsilon}\right\|_{\Omega^{\varepsilon}}^{2} \leq C_{5}\left\|v^{\varepsilon}\right\|_{\Omega^{\varepsilon}}^{2}+K
$$

Integrating with respect to time, we obtain

Hence

$$
\left\|v^{\varepsilon}(t)\right\|_{\Omega^{\varepsilon}}^{2}+\frac{D}{2}\left\|\nabla v^{\varepsilon}\right\|_{\Omega^{\varepsilon}, t}^{2} \leq C .
$$

and

$$
\left\|v^{\varepsilon}(t)\right\|_{\Omega^{\varepsilon}}^{2} \leq C
$$

In a similar manner, we can get

$$
\left\|\nabla v^{\varepsilon}(t)\right\|_{\Omega^{\varepsilon}, t}^{2} \leq C
$$

$$
\left\|\frac{\partial v^{\varepsilon}}{\partial t}(t)\right\|_{\Omega^{\varepsilon}}^{2} \leq C .
$$




\subsection{Limit passage}

The remaining step in the proof of Theorem 2.5 will be divided into four new steps.

First step. Let $v^{\varepsilon} \in \mathcal{W}_{0}\left(\Omega^{\varepsilon}\right)$ be the solution of the variational problem (2.8) and let $P^{\varepsilon} v^{\varepsilon}$ be the extension of $v^{\varepsilon}$ inside the holes given by Lemma 2.2. Using our a priori estimates (3.5)-(3.6), we easily can see that there exists a constant $C$ depending on $T$ and the data, but independent of $\varepsilon$ such that

$$
\left\|P^{\varepsilon} v^{\varepsilon}(t)\right\|_{\Omega}+\left\|\nabla P^{\varepsilon} v^{\varepsilon}\right\|_{\Omega, t}+\left\|\partial_{t}\left(P^{\varepsilon} v^{\varepsilon}\right)(t)\right\|_{\Omega} \leq C
$$

for all $t \leq T$. Consequently, by passing to a subsequence, still denoted by $P^{\varepsilon} v^{\varepsilon}$, we can assume that there exists $v \in \mathcal{V}$ such that the following convergence properties hold:

$$
\begin{gathered}
P^{\varepsilon} v^{\varepsilon} \rightarrow v \quad \text { weakly in } \mathcal{V}, \\
\partial_{t}\left(P^{\varepsilon} v^{\varepsilon}\right) \rightarrow \partial_{t} v \quad \text { weakly in } \mathcal{H}, \\
P^{\varepsilon} v^{\varepsilon} \rightarrow v \quad \text { strongly in } \mathcal{H} .
\end{gathered}
$$

It remains to identify the limit equation satisfied by $v$.

Second step. In order to get the limit equation satisfied by $v$ we have to pass to the limit in (2.8). For getting the limit of the second term in the left hand side of (2.8), let us introduce, for any $h \in L^{s^{\prime}}(\partial T), 1 \leq s^{\prime} \leq \infty$, the linear form $\mu_{h}^{\varepsilon}$ on $W_{0}^{1, s}(\Omega)$ defined by

$$
\left\langle\mu_{h}^{\varepsilon}, \varphi\right\rangle=\varepsilon \int_{S^{\varepsilon}} h\left(\frac{x}{\varepsilon}\right) \varphi d \sigma \quad \forall \varphi \in W_{0}^{1, s}(\Omega),
$$

with $1 / s+1 / s^{\prime}=1$. It is proved in [8] that

$$
\mu_{h}^{\varepsilon} \rightarrow \mu_{h} \quad \text { strongly in }\left(W_{0}^{1, s}(\Omega)\right)^{\prime},
$$

where

$$
\left\langle\mu_{h}, \varphi\right\rangle=\mu_{h} \int_{\Omega} \varphi d x
$$

with

$$
\mu_{h}=\frac{1}{|Y|} \int_{\partial F} h(y) d \sigma .
$$

In the particular case in which $h \in L^{\infty}(\partial F)$ or even $h$ is constant, we have

$$
\mu_{h}^{\varepsilon} \rightarrow \mu_{h} \quad \text { strongly in } W^{-1, \infty}(\Omega) .
$$

In what follows, we shall denote by $\mu^{\varepsilon}$ the above introduced measure in the particular case in which $h=1$. Notice that in this case $\mu_{h}$ becomes $\mu_{1}=\frac{|\partial F|}{|Y|}$.

Moreover, if $z^{\varepsilon} \in H_{0}^{1}(\Omega)$ is such that $z^{\varepsilon} \rightarrow z$ weakly in $H_{0}^{1}(\Omega)$, then (see [9])

$$
\left\langle\mu_{h}^{\varepsilon},\left.z^{\varepsilon}\right|_{\Omega^{\varepsilon}}\right\rangle \rightarrow \mu_{h} \int_{\Omega} z d x .
$$

Let us prove now that for any $\varphi \in C_{0}^{\infty}(\Omega)$ and for any $z^{\varepsilon} \rightarrow z$ weakly in $H_{0}^{1}(\Omega)$, we get

$$
\varphi g\left(z^{\varepsilon}\right) \rightarrow \varphi g(z) \quad \text { weakly in } W_{0}^{1, \bar{q}}(\Omega)
$$


where

$$
\bar{q}=\frac{2 n}{q(n-2)+n} .
$$

To prove (3.18), let us first note that

$$
\sup \left\|\nabla g\left(z^{\varepsilon}\right)\right\|_{L^{\bar{q}}(\Omega)}<\infty .
$$

Indeed, from the growth condition (2.7) imposed to $g$, we get

$$
\begin{gathered}
\int_{\Omega}\left|\frac{\partial g}{\partial x_{i}}\left(z^{\varepsilon}\right)\right|^{\bar{q}} d x \leq C \int_{\Omega}\left(1+\left|z^{\varepsilon}\right|^{q \bar{q}}\right)\left|\frac{\partial z^{\varepsilon}}{\partial x_{i}}\right|^{\bar{q}} d x \leq \\
\leq C\left(1+\left(\int_{\Omega}\left|z^{\varepsilon}\right|^{q \bar{q} \gamma} d x\right)^{1 / \gamma}\right)\left(\int_{\Omega}\left|\nabla z^{\varepsilon}\right|^{\bar{q} \delta} d x\right)^{1 / \delta},
\end{gathered}
$$

where we took $\gamma$ and $\delta$ such that $\bar{q} \delta=2,1 / \gamma+1 / \delta=1$ and $q \bar{q} \gamma=2 n /(n-2)$. Notice that, since $0 \leq q<n /(n-2)$, we have $\bar{q}>1$. Now, since

$$
\sup \left\|z^{\varepsilon}\right\|_{L^{\frac{2 n}{n-2}}(\Omega)}<\infty
$$

we get immediately (3.19). Hence, to get (3.18), it remains only to prove that

$$
g\left(z^{\varepsilon}\right) \rightarrow g(z) \quad \text { strongly in } L^{\bar{q}}(\Omega) .
$$

But this is just a consequence of the following well-known result (see [20]):

Theorem 3.3 Let $G: \Omega \times \mathbb{R} \rightarrow \mathbb{R}$ be a Carathéodory function, i.e.

a) for every $z$ the function $G(\cdot, z)$ is measurable with respect to $x \in \Omega$.

b) for every (a.e.) $x \in \Omega$, the function $G(x, \cdot)$ is continuous with respect to $z$.

Moreover, if we assume that there exists a positive constant $C$ such that

$$
|G(x, z)| \leq C\left(1+|z|^{r / t}\right)
$$

with $r \geq 1$ and $t<\infty$, then the map $z \in L^{r}(\Omega) \mapsto G(x, z(x)) \in L^{t}(\Omega)$ is continuous in the strong topologies.

Indeed, since

$$
|g(z)| \leq C\left(1+|z|^{q+1}\right)
$$

applying the above theorem for $G(x, z)=g(z), t=\bar{q}$ and $r=(2 n /(n-2))-r^{\prime}$, with $r^{\prime}>0$ such that $q+1<r / t$ and using the compact injection $H^{1}(\Omega) \hookrightarrow L^{r}(\Omega)$ we easily get (3.20).

Finally, from (3.16) (with $h=1$ ) and (3.18) written for $z^{\varepsilon}=P^{\varepsilon} v^{\varepsilon}(t)$, we conclude

$$
\left\langle\mu^{\varepsilon}, \varphi g\left(P^{\varepsilon} v^{\varepsilon}(t)\right)\right\rangle \rightarrow \frac{|\partial F|}{|Y|} \int_{\Omega} \varphi g(v(t)) d x \quad \forall \varphi \in \mathcal{D} .
$$

We are now in a position to use Lebesgue's convergence theorem. To this end, we use the above pointwise convergence, the a priori estimates (3.5) and the growth condition (2.7). As a result, we get

$$
\lim _{\varepsilon \rightarrow 0} \varepsilon \gamma\left(g\left(v^{\varepsilon}\right), \varphi\right)_{S^{\varepsilon}, T}=\frac{|\partial F|}{|Y|} \gamma(g(v), \varphi)_{\Omega, T}
$$


which is the desired result. This ends the second step of the proof.

Third step. Let $\xi^{\varepsilon}$ be the gradient of $v^{\varepsilon}$ in $\Omega^{\varepsilon}$ and let us denote by $\widetilde{\xi^{\varepsilon}}$ its extension with zero to the whole of $\Omega$, i.e.

$$
\widetilde{\xi^{\varepsilon}}=\left\{\begin{array}{llc}
\xi^{\varepsilon} & \text { in } & \Omega^{\varepsilon}, \\
0 & \text { in } & \Omega \backslash \overline{\Omega^{\varepsilon}} .
\end{array}\right.
$$

Obviously, $\widetilde{\xi^{\varepsilon}}$ is bounded in $(\mathcal{H}(\Omega))^{n}$ and hence there exists $\xi \in(\mathcal{H}(\Omega))^{n}$ such that

$$
\widetilde{\xi^{\varepsilon}} \rightarrow \xi \quad \text { weakly in }(\mathcal{H}(\Omega))^{n} .
$$

Let us see now which is the equation satisfied by $\xi$. Take $\varphi \in \mathcal{D}$. From (2.8) we get

$$
-\left(\chi_{\Omega^{\varepsilon}} P^{\varepsilon} v^{\varepsilon}, \frac{d \varphi}{d t}\right)_{\Omega, T}+D\left(\widetilde{\xi^{\varepsilon}}, \nabla \varphi\right)_{\Omega, T}+\varepsilon\left(f^{\varepsilon}, \varphi\right)_{S^{\varepsilon}, T}=\left(\chi_{\Omega^{\varepsilon}} h, \varphi\right)_{\Omega, T} .
$$

Now, we can pass to the limit, with $\varepsilon \rightarrow 0$, in all the terms of (3.24). For the first one, we have

$$
-\lim _{\varepsilon \rightarrow 0}\left(\chi_{\Omega^{\varepsilon}} P^{\varepsilon} v^{\varepsilon}, \frac{d \varphi}{d t}\right)_{\Omega, T}=-\frac{\left|Y^{*}\right|}{|Y|}\left(v, \frac{d \varphi}{d t}\right)_{\Omega, T}
$$

For the second term we get

$$
\lim _{\varepsilon \rightarrow 0} D\left(\widetilde{\xi^{\varepsilon}}, \nabla \varphi\right)_{\Omega, T}=D(\xi, \nabla \varphi)_{\Omega, T} .
$$

To get the limit of the third term, let us notice that using (3.22), we get

$$
\lim _{\varepsilon \rightarrow 0} \varepsilon\left(g\left(v^{\varepsilon}\right), \varphi\right)_{S^{\varepsilon}, T}=\frac{|\partial F|}{|Y|}(g(v), \varphi)_{\Omega, T} .
$$

Let us prove now that

$$
\lim _{\varepsilon \rightarrow 0} \varepsilon\left(w^{\varepsilon}, \varphi\right)_{S^{\varepsilon}, T}=\frac{|\partial F|}{|Y|}(\gamma r \star g(v), \varphi)_{\Omega, T}, \quad \forall \varphi \in \mathcal{D} .
$$

Indeed, we have

$$
\begin{gathered}
\varepsilon\left(w^{\varepsilon}, \varphi\right)_{S^{\varepsilon}, T}=\varepsilon \int_{0}^{T} \int_{S^{\varepsilon}} w^{\varepsilon}(t, x) \varphi(t, x) d x d t= \\
=\varepsilon \int_{0}^{T} \int_{S^{\varepsilon}}\left(w_{1}(x) e^{-(a+\gamma) t}+\int_{0}^{t} \gamma g\left(v^{\varepsilon}(s, x)\right) e^{-(a+\gamma)(t-s)} d s\right) \varphi(t, x) d x d t= \\
=\int_{0}^{T} e^{-(a+\gamma) t}\left[\varepsilon \int_{S^{\varepsilon}} w_{1}(x) \varphi(t, x) d x\right] d t+\int_{0}^{T}\left[\int_{0}^{t} \gamma e^{-(a+\gamma)(t-s)}\left(\varepsilon \int_{S^{\varepsilon}} g\left(v^{\varepsilon}(s, x)\right) \varphi(t, x) d x\right) d s\right] d t .
\end{gathered}
$$

Therefore

$$
\begin{aligned}
& \lim _{\varepsilon \rightarrow 0} \varepsilon\left(w^{\varepsilon}, \varphi\right)_{S^{\varepsilon}, T}=\lim _{\varepsilon \rightarrow 0}\left\{\int_{0}^{T} e^{-(a+\gamma) t}\left[\varepsilon \int_{S^{\varepsilon}} w_{1}(x) \varphi(t, x) d x\right] d t+\right. \\
& \left.\quad+\int_{0}^{T}\left[\int_{0}^{t} \gamma e^{-(a+\gamma)(t-s)}\left(\varepsilon \int_{S^{\varepsilon}} g\left(v^{\varepsilon}(s, x)\right) \varphi(t, x) d x\right) d s\right] d t\right\}=
\end{aligned}
$$




$$
\begin{gathered}
\left.=\int_{0}^{T} e^{-(a+\gamma) t}\left[\frac{|\partial F|}{|Y|} \int_{\Omega} w_{1}(x) \varphi(t, x) d x\right] d t+\int_{0}^{T} \int_{0}^{t} \gamma e^{-(a+\gamma)(t-s)}\left(\frac{|\partial F|}{|Y|} \int_{\Omega} g(v(s, x)) \varphi(t, x) d x\right) d s\right] d t= \\
=\int_{0}^{T} e^{-(a+\gamma) t}\left[\frac{|\partial F|}{|Y|} \int_{\Omega} w_{1}(x) \varphi(t, x) d x\right] d t+\int_{0}^{T} \int_{\Omega} \frac{|\partial F|}{|Y|} \gamma\left[\int_{0}^{t} e^{-(a+\gamma)(t-s)} g(v(s, x)) d s\right] \varphi(t, x) d x d t= \\
=\frac{|\partial F|}{|Y|} \int_{0}^{T}\left\{\int_{\Omega}\left[w_{1}(x) e^{-(a+\gamma) t}+\gamma r \star g(v(\cdot, x))(t)\right] \varphi(t, x) d x\right\} d t .
\end{gathered}
$$

From (3.27) and (3.28) we get

$$
\lim _{\varepsilon \rightarrow 0} \varepsilon\left(f^{\varepsilon}, \varphi\right)_{S^{\varepsilon}, T}=\frac{\left|Y^{\star}\right|}{|Y|}\left(F_{0}, \varphi\right)_{\Omega, T}, \quad \forall \varphi \in \mathcal{D} .
$$

It is not difficult to pass to the limit in the right-hand side of (3.24). Since

$$
\chi_{\Omega^{\varepsilon}} h \rightarrow \frac{\left|Y^{*}\right|}{|Y|} h \quad \text { weakly in } \mathcal{H}(\Omega)
$$

we obtain

$$
\lim _{\varepsilon \rightarrow 0}\left(\chi_{\Omega^{\varepsilon}} h, \varphi\right)_{\Omega, T}=\frac{\left|Y^{*}\right|}{|Y|}(h, \varphi)_{\Omega, T} .
$$

Putting together (3.25)-(3.26) and (3.29)-(3.30), we have

$$
-\frac{\left|Y^{\star}\right|}{|Y|}\left(v, \frac{d \varphi}{d t}\right)_{\Omega, T}+D(\xi, \nabla \varphi)_{\Omega, T}+\frac{\left|Y^{\star}\right|}{|Y|}\left(F_{0}, \varphi\right)_{\Omega, T}=\frac{\left|Y^{*}\right|}{|Y|}(h, \varphi)_{\Omega, T} \quad \forall \varphi \in \mathcal{D}(\Omega) .
$$

Hence $\xi$ verifies

$$
\frac{\left|Y^{*}\right|}{|Y|} \frac{\partial v}{\partial t}-D \operatorname{div} \xi+\frac{\left|Y^{*}\right|}{|Y|} F_{0}=\frac{\left|Y^{*}\right|}{|Y|} h, \quad t>0, x \in \Omega
$$

It remains now to identify $\xi$.

Fourth step. In order to identify $\xi$, we shall make use of the solutions of the cell-problems (2.15). For any fixed $i=1, \ldots, n$, let us define

$$
\Phi_{i \varepsilon}(x)=\varepsilon\left(\chi_{i}\left(\frac{x}{\varepsilon}\right)+y_{i}\right) \quad \forall x \in \Omega^{\varepsilon},
$$

where

$$
y=\frac{x}{\varepsilon} .
$$

By periodicity

$$
P^{\varepsilon} \Phi_{i \varepsilon} \rightarrow x_{i} \quad \text { weakly in } H^{1}(\Omega) .
$$

Let $\eta_{i}^{\varepsilon}$ be the gradient of $\Phi_{i \varepsilon}$ in $\Omega^{\varepsilon}$. Denote by $\widetilde{\eta_{i}^{\varepsilon}}$ the extension by zero of $\eta_{i}^{\varepsilon}$ inside the holes. From (3.32), for the $j$-component of $\widetilde{\eta_{i}^{\varepsilon}}$ we get

$$
\left(\widetilde{\eta_{i}^{\varepsilon}}\right)_{j}=\left(\widetilde{\frac{\partial \Phi_{i \varepsilon}}{\partial x_{j}}}\right)=\left(\widetilde{\frac{\partial \chi_{i}}{\partial y_{j}}}(y)\right)+\delta_{i j} \chi_{Y^{*}}
$$


and hence

$$
\left(\widetilde{\eta_{i}^{\varepsilon}}\right)_{j} \rightarrow \frac{1}{|Y|}\left(\int_{Y^{*}} \frac{\partial \chi_{i}}{\partial y_{j}} d y+\left|Y^{*}\right| \delta_{i j}\right)=\frac{\left|Y^{*}\right|}{|Y|} q_{i j} \quad \text { weakly in } L^{2}(\Omega) .
$$

On the other hand, it is not difficult to see that $\eta_{i}^{\varepsilon}$ satisfies

$$
\left\{\begin{array}{l}
-\operatorname{div} \eta_{i}^{\varepsilon}=0 \quad \text { in } \Omega^{\varepsilon} \\
\eta_{i}^{\varepsilon} \cdot \nu=0 \quad \text { on } S^{\varepsilon}
\end{array}\right.
$$

Now, let $\varphi \in \mathcal{D}$. Multiplying the first equation in (3.35) by $\varphi v^{\varepsilon}$ and integrating by parts over $\Omega^{\varepsilon}$ we get

$$
\left(\eta_{i}^{\varepsilon}, \nabla \varphi v^{\varepsilon}\right)_{\Omega^{\varepsilon}, T}+\left(\eta_{i}^{\varepsilon}, \nabla v^{\varepsilon} \varphi\right)_{\Omega^{\varepsilon}, T}=0
$$

So

$$
\left(\widetilde{\eta_{i}^{\varepsilon}}, \nabla \varphi P^{\varepsilon} v^{\varepsilon}\right)_{\Omega, T}+\left(\eta_{i}^{\varepsilon}, \nabla v^{\varepsilon} \varphi\right)_{\Omega^{\varepsilon}, T}=0 .
$$

On the other hand, taking $\varphi \Phi_{i \varepsilon}$ as a test function in (2.8) we obtain

$$
-\left(v^{\varepsilon}, \Phi_{i \varepsilon} \frac{d \varphi}{d t}\right)_{\Omega^{\varepsilon}, T}+D\left(\nabla v^{\varepsilon}, \nabla \varphi \Phi_{i \varepsilon}\right)_{\Omega^{\varepsilon}, T}+D\left(\nabla v^{\varepsilon}, \nabla \Phi_{i \varepsilon} \varphi\right)_{\Omega^{\varepsilon}, T}+\varepsilon\left(f^{\varepsilon}, \varphi \Phi_{i \varepsilon}\right)_{S^{\varepsilon}, T}=\left(h, \varphi \Phi_{i \varepsilon}\right)_{\Omega^{\varepsilon}, T},
$$

which, using the definitions of $\widetilde{\xi}$ and $\widetilde{\eta_{i}^{\varepsilon}}$, gives

$$
\begin{gathered}
-\left(\chi_{\Omega^{\varepsilon}} P^{\varepsilon} v^{\varepsilon}, P^{\varepsilon} \Phi_{i \varepsilon} \frac{d \varphi}{d t}\right)_{\Omega, T}+D\left(\widetilde{\xi^{\varepsilon}}, \nabla \varphi P^{\varepsilon} \Phi_{i \varepsilon}\right)_{\Omega, T}+D\left(\nabla v^{\varepsilon}, \eta_{i}^{\varepsilon} \varphi\right)_{\Omega^{\varepsilon}, T}+ \\
+\varepsilon\left(f^{\varepsilon}, \varphi \Phi_{i \varepsilon}\right)_{S^{\varepsilon}, T}=\left(h \chi_{\Omega^{\varepsilon}}, \varphi P^{\varepsilon} \Phi_{i \varepsilon}\right)_{\Omega, T} .
\end{gathered}
$$

Now, using (3.36), we get

$$
\begin{gathered}
-\left(\chi_{\Omega^{\varepsilon}} P^{\varepsilon} v^{\varepsilon}, P^{\varepsilon} \Phi_{i \varepsilon} \frac{d \varphi}{d t}\right)_{\Omega, T}+D\left(\widetilde{\xi^{\varepsilon}}, \nabla \varphi P^{\varepsilon} \Phi_{i \varepsilon}\right)_{\Omega, T}-D\left(\widetilde{\eta_{i}^{\varepsilon}}, \nabla \varphi P^{\varepsilon} \Phi_{i \varepsilon}\right)_{\Omega, T}+ \\
+\varepsilon\left(f^{\varepsilon}, \varphi \Phi_{i \varepsilon}\right)_{S^{\varepsilon}, T}=\left(h \chi_{\Omega^{\varepsilon}}, \varphi P^{\varepsilon} \Phi_{i \varepsilon}\right)_{\Omega, T} .
\end{gathered}
$$

Let us pass to the limit in (3.37). For the first term we obviously have

$$
-\lim _{\varepsilon \rightarrow 0}\left(\chi_{\Omega^{\varepsilon}} P^{\varepsilon} v^{\varepsilon}, P^{\varepsilon} \Phi_{i \varepsilon} \frac{d \varphi}{d t}\right)_{\Omega, T}=-\frac{\left|Y^{*}\right|}{|Y|}\left(v, x_{i} \frac{d \varphi}{d t}\right)_{\Omega, T} .
$$

For the second one, using (3.23) and (3.33), we have

$$
\lim _{\varepsilon \rightarrow 0}\left(\widetilde{\xi}, \nabla \varphi P^{\varepsilon} \Phi_{i \varepsilon}\right)_{\Omega, T}=\left(\xi, \nabla \varphi x_{i}\right)_{\Omega, T} .
$$

On the other hand, (3.15) and (3.34) imply that

$$
\lim _{\varepsilon \rightarrow 0}\left(\widetilde{\eta_{i}^{\varepsilon}}, \nabla \varphi P^{\varepsilon} v^{\varepsilon}\right)_{\Omega, T}=\frac{\left|Y^{*}\right|}{|Y|}\left(q_{i}, \nabla \varphi v\right)_{\Omega, T}
$$

where $q_{i}$ is the vector having the $j$-component equal to $q_{i j}$. 
Because the boundary of $F$ is smooth, of class $C^{2}, P^{\varepsilon} \Phi_{i \varepsilon} \in W^{1, \infty}(\Omega)$ and $P^{\varepsilon} \Phi_{i \varepsilon} \rightarrow x_{i}$ strongly in $L^{\infty}(\Omega)$. Then

$$
\lim _{\varepsilon \rightarrow 0} \varepsilon\left(g\left(v^{\varepsilon}\right), \varphi \Phi_{i \varepsilon}\right)_{S^{\varepsilon}, T}=\frac{|\partial F|}{|Y|}\left(g(v), \varphi x_{i}\right)_{\Omega, T}
$$

and hence

$$
\lim _{\varepsilon \rightarrow 0} \varepsilon\left(f^{\varepsilon}, \varphi \Phi_{i \varepsilon}\right)_{S^{\varepsilon}, T}=\frac{\left|Y^{\star}\right|}{|Y|}\left(F_{0}, \varphi x_{i}\right)_{\Omega, T}
$$

Finally, for the limit of the right-hand side of (3.37), since $\chi_{\Omega^{\varepsilon}} h \rightarrow \frac{\left|Y^{*}\right|}{|Y|} h$ weakly in $\mathcal{H}(\Omega)$, using again (3.33) we have

$$
\lim _{\varepsilon \rightarrow 0}\left(h \chi_{\Omega^{\varepsilon}}, \varphi P^{\varepsilon} \Phi_{i \varepsilon}\right)_{\Omega, T}=\frac{\left|Y^{*}\right|}{|Y|}\left(h, \varphi x_{i}\right)_{\Omega, T}
$$

Hence we get

$$
\begin{gathered}
-\frac{\left|Y^{*}\right|}{|Y|}\left(v, x_{i} \frac{d \varphi}{d t}\right)_{\Omega, T}+D\left(\xi, \nabla \varphi x_{i}\right)_{\Omega, T}-D \frac{\left|Y^{*}\right|}{|Y|}\left(q_{i}, \nabla \varphi v\right)_{\Omega, T}+ \\
+\frac{\left|Y^{\star}\right|}{|Y|}\left(F_{0}, \varphi x_{i}\right)_{\Omega, T}=\frac{\left|Y^{*}\right|}{|Y|}\left(h, \varphi x_{i}\right)_{\Omega, T} .
\end{gathered}
$$

Using Green's formula and equation (3.31), we have

$$
-D\left(\xi, \nabla x_{i} \varphi\right)_{\Omega, T}+D \frac{\left|Y^{*}\right|}{|Y|}\left(q_{i}, \nabla v \varphi\right)_{\Omega, T}=0 .
$$

The above equality holds true for any $\varphi \in \mathcal{D}(\Omega)$. This implies that

$$
-D \xi \cdot \nabla x_{i}+D \frac{\left|Y^{*}\right|}{|Y|} q_{i} \cdot \nabla v=0, \quad t>0, x \in \Omega
$$

Writing (3.45) by components, derivating with respect to $x_{i}$, summing after $i$ and using (3.31), we conclude that

$$
D \frac{\left|Y^{*}\right|}{|Y|} \sum_{i, j=1}^{n} q_{i j} \frac{\partial^{2} v}{\partial x_{i} \partial x_{j}}=D \operatorname{div} \xi=\frac{\left|Y^{*}\right|}{|Y|} \frac{\partial v}{\partial t}+\frac{\left|Y^{*}\right|}{|Y|} F_{0}-\frac{\left|Y^{*}\right|}{|Y|} h,
$$

which means that $v$ satisfies

$$
\frac{\partial v}{\partial t}-D \sum_{i, j=1}^{n} q_{i j} \frac{\partial^{2} v}{\partial x_{i} \partial x_{j}}+F_{0}(t, x)=h, \quad t>0, x \in \Omega .
$$

Since $v \in \mathcal{W}_{0}(\Omega)$ (i.e. $v=0$ on $\partial \Omega$ ) and $v$ is uniquely determined, the whole sequence $P^{\varepsilon} v^{\varepsilon}$ converges to $v$ and Theorem 2.5 is proved.

\subsection{The case of a non-smooth boundary condition}

In this subsection we want to cover Example b) in the Introduction, namely the case in which the function $g$ appearing in (1.4) is given by

$$
g(v)=|v|^{p-1} v, \quad 0<p<1 \quad \text { (Freundlich kinetics) }
$$


For this case, we see that $g$ is a single-valued maximal monotone graph in $\mathbb{R} \times \mathbb{R}$, satisfying the condition $g(0)=0$. Also, if we denote by $D(g)$ the domain of $g$, i.e. $D(g)=\{\xi \in \mathbb{R} \mid g(\xi) \neq \varnothing\}$, then $D(g)=\mathbb{R}$. Moreover, $g$ is continuous and satisfies

$$
|g(v)| \leq C(1+|v|)
$$

We know that in this case there exists a lower semicontinuous convex function $G$ from $\mathbb{R}$ to ] $-\infty,+\infty$ ], $G$ proper, i.e. $G \not \equiv+\infty$ such that $g$ is the subdifferential of $G, g=\partial G$ ( $G$ is an indefinite "integral" of $g$ ).

The main result of this section is the following one:

Theorem 3.4 One can construct an extension $P^{\varepsilon} v^{\varepsilon}$ of the solution $v^{\varepsilon}$ of the problem $\left(V^{\varepsilon}\right)$ such that

$$
P^{\varepsilon} v^{\varepsilon} \rightarrow v \quad \text { weakly in } \mathcal{V}
$$

where $v$ is the unique solution of the following limit problem:

$$
\left\{\begin{array}{l}
\frac{\partial v}{\partial t}(t, x)+F_{0}(t, x)-D \sum_{i, j=1}^{n} q_{i j} \frac{\partial^{2} v}{\partial x_{i} \partial x_{j}}(t, x)=h(t, x), \quad t>0, x \in \Omega \\
v(t, x)=0, \quad t>0, x \in \partial \Omega \\
v(t, x)=v_{1}(x), \quad t=0, x \in \Omega
\end{array}\right.
$$

with

$$
F_{0}(t, x)=\frac{|\partial F|}{\left|Y^{\star}\right|} \gamma\left[g(v(t, x))-w_{1}(x) e^{-(a+\gamma) t}-\gamma r(\cdot) \star g(v(\cdot, x))(t)\right] .
$$

In (3.46), $Q=\left(\left(q_{i j}\right)\right)$ is the classical homogenized matrix, whose entries were defined by (2.14)(2.15). Moreover, the limit problem for the surface concentration is:

$$
\left\{\begin{array}{l}
\frac{\partial w}{\partial t}(t, x)+(a+\gamma) w(t, x)=\gamma g(v(t, x)), \quad t>0, x \in \Omega \\
w(t, x)=w_{1}(x), \quad t=0, x \in \Omega
\end{array}\right.
$$

and obviously

$$
w(t, x)=w_{1}(x) e^{-(a+\gamma) t}+\gamma r(t) \star g(v(t, x)) . \quad \text { - }
$$

Proof. To deal with this case, we can use an approximation technique, namely Yosida regularization technique. Let $\lambda>0$ be given. We consider the approximating problems:

$$
\left\{\begin{array}{l}
\text { Find } v_{\lambda}^{\varepsilon} \in \mathcal{W}_{0}\left(\Omega^{\varepsilon}\right), v_{\lambda}^{\varepsilon}(0)=\left.v_{1}\right|_{\Omega^{\varepsilon}} \text { such that } \\
-\left(v_{\lambda}^{\varepsilon}, \frac{d \varphi}{d t}\right)_{\Omega^{\varepsilon}, T}+\varepsilon\left(f_{\lambda}^{\varepsilon}, \varphi\right)_{\Omega^{\varepsilon}, T}=-D\left(\nabla v_{\lambda}^{\varepsilon}, \nabla \varphi\right)_{\Omega^{\varepsilon}, T}+(h, \varphi)_{\Omega^{\varepsilon}, T}, \quad \forall \varphi \in \mathcal{W}_{0}\left(\Omega^{\varepsilon}\right)
\end{array}\right.
$$

and

$$
\left\{\begin{array}{l}
\text { Find } w_{\lambda}^{\varepsilon} \in \mathcal{W}\left(S^{\varepsilon}\right), w_{\lambda}^{\varepsilon}(0)=\left.w_{1}\right|_{S^{\varepsilon}} \text { such that } \\
-\left(w_{\lambda}^{\varepsilon}, \frac{d \varphi}{d t}\right)_{S^{\varepsilon}, T}+a\left(w_{\lambda}^{\varepsilon}, \varphi\right)_{S^{\varepsilon}, T}=\left(f_{\lambda}^{\varepsilon}, \varphi\right)_{S^{\varepsilon}, T}, \quad \forall \varphi \in \mathcal{W}\left(S^{\varepsilon}\right)
\end{array}\right.
$$

where

$$
f_{\lambda}^{\varepsilon}=\gamma\left(g_{\lambda}\left(v_{\lambda}^{\varepsilon}\right)-w_{\lambda}^{\varepsilon}\right)
$$

and

$$
g_{\lambda}=\frac{I-J_{\lambda}}{\lambda}
$$


with

$$
J_{\lambda}=(I+\lambda \partial G)^{-1} .
$$

Note that $g_{\lambda}$ is a Lipschitz function, which is non-decreasing and satisfies the condition $g_{\lambda}(0)=0$.

Problem (3.49)-(3.50) has a unique solution $\left(v_{\lambda}^{\varepsilon}, w_{\lambda}^{\varepsilon}\right)$, for every $\lambda>0$ (see [7] and [20]). As we saw in Chapter 2, we can express $w_{\lambda}^{\varepsilon}$ in terms of $v_{\lambda}^{\varepsilon}$; therefore, it is enough to get a problem only for $v_{\lambda}^{\varepsilon}$ and in what follows we shall focus our attention only on getting the limit problem for $v_{\lambda}^{\varepsilon}$.

Mollifying $g_{\lambda}$ to make it a smooth function (see [5]) and using the results of the previous chapter, for any $\lambda>0$, we get

$$
P^{\varepsilon} v_{\lambda}^{\varepsilon} \rightarrow v_{\lambda} \quad \text { strongly in } \mathcal{H}(\Omega) .
$$

Then, it is not difficult to see that, proving suitable a priori estimates (classical energy estimates) on the solutions $v_{\lambda}$, we can ensure, via compactness arguments (see [3]), the strong convergence of $v_{\lambda}$, as $\lambda \rightarrow 0$, to $v$, the unique solution of problem (3.46). Hence

$$
v_{\lambda} \rightarrow v \quad \text { strongly in } \mathcal{H}(\Omega) \text {. }
$$

Finally, since

$$
\left\|P^{\varepsilon} v^{\varepsilon}-v\right\|_{\Omega, T} \leq\left\|P^{\varepsilon} v^{\varepsilon}-P^{\varepsilon} v_{\lambda}^{\varepsilon}\right\|_{\Omega, T}+\left\|P^{\varepsilon} v_{\lambda}^{\varepsilon}-v_{\lambda}\right\|_{\Omega, T}+\left\|v_{\lambda}-v\right\|_{\Omega, T},
$$

we get the strong convergence of $P^{\varepsilon} v^{\varepsilon}$ to $v$ in $\mathcal{H}(\Omega)$.

Remark 3.5 The conclusion of the above theorem remains true for more general situations. It is the case of the so-called zeroth-order reactions, in which, formally, $g$ is given by the discontinuous function $g(v)=0$, if $v \leq 0$ and $g(v)=1$ if $v>0$ (see, for instance, [2]). The correct mathematical treatment needs the problem to be reformulated by using the maximal monotone graph of $\mathbb{R}^{2}$ associated to the Heaviside function $\beta(v)=\{0\}$ if $v<0, \beta(0)=[0,1]$ and $\beta(v)=\{1\}$ if $v>0$. The existence and uniqueness of a solution can be found, for instance, in H. BRÉzIS [7]. The solution is obtained by passing to the limit in a sequence of problems associated to a monotone sequence of Lipschitz functions approximating $\beta$ and so the results of this section remain true.

\section{Laplace-Beltrami model with oscillating coefficients}

In a similar manner we can treat the case in which the surface $\partial F$ is physically and chemically heterogeneous and more precisely, the case in which the reaction and the adsorption coefficients $a$ and $\gamma$, respectively, are rapidly oscillating functions, i.e.

$$
a^{\varepsilon}(x)=a\left(\frac{x}{\varepsilon}\right), \gamma^{\varepsilon}(x)=\gamma\left(\frac{x}{\varepsilon}\right),
$$

with $a$ and $\gamma$ positive functions in $W^{1, \infty}(\Omega)$ which are $Y$-periodic (for linear adsorption rates, see [19]).

In this case, $v^{\varepsilon}$ and $w^{\varepsilon}$ satisfy the following system of equations:

$$
\begin{gathered}
\left(\bar{V}^{\varepsilon}\right)\left\{\begin{array}{l}
\frac{\partial v^{\varepsilon}}{\partial t}(t, x)-D \Delta v^{\varepsilon}(t, x)=h(t, x), \quad x \in \Omega^{\varepsilon}, t>0, \\
v^{\varepsilon}(t, x)=0, \quad x \in \partial \Omega, t>0 \\
v^{\varepsilon}(t, x)=v_{1}(x), \quad x \in \Omega^{\varepsilon}, t=0
\end{array}\right. \\
-D \frac{\partial v^{\varepsilon}}{\partial \nu}(t, x)=\varepsilon f^{\varepsilon}(t, x), \quad x \in S^{\varepsilon}, t>0
\end{gathered}
$$


and

$$
\left(\bar{W}^{\varepsilon}\right)\left\{\begin{array}{l}
\frac{\partial w^{\varepsilon}}{\partial t}(t, x)+a^{\varepsilon}(x) w^{\varepsilon}(t, x)=f^{\varepsilon}(t, x), \quad x \in S^{\varepsilon}, t>0, \\
w^{\varepsilon}(t, x)=w_{1}(x), \quad x \in S^{\varepsilon}, t=0
\end{array}\right.
$$

where

$$
f^{\varepsilon}(t, x)=\gamma^{\varepsilon}(x)\left(g\left(v^{\varepsilon}(t, x)\right)-w^{\varepsilon}(t, x)\right)
$$

Denote

$$
y=\frac{x}{\varepsilon} .
$$

The main result of this section is the following one:

Theorem 4.1 The effective behavior of $v$ and $w$ is governed by the following system:

$$
\left\{\begin{array}{l}
\frac{\partial v}{\partial t}(t, x)+G_{0}(t, x)-D \sum_{i, j=1}^{n} q_{i j} \frac{\partial^{2} v}{\partial x_{i} \partial x_{j}}=h(t, x), \quad t>0, x \in \Omega, \\
v(t, x)=0 \quad t>0, x \in \partial \Omega \\
v(t, x)=v_{1}(x) \quad t=0, x \in \Omega
\end{array}\right.
$$

and

$$
\left\{\begin{array}{l}
\frac{\partial w}{\partial t}(t, x, y)+(a(y)+\gamma(y)) w(t, x, y)=\gamma(y) g(v(t, x)), \quad t>0, x \in \Omega, y \in \partial F \\
w(t, x, y)=w_{1}(x) \quad t=0, x \in \Omega, y \in \partial F
\end{array}\right.
$$

where

$$
G_{0}(t, x)=\frac{1}{\left|Y^{\star}\right|} \int_{\partial F} f_{0}(t, x, y) d \sigma
$$

and

$$
f_{0}=\gamma(y)(g(v(t, x))-w(t, x, y))
$$

In (4.5), $Q=\left(\left(q_{i j}\right)\right)$ is the classical homogenized matrix, whose entries were defined by (2.14)(2.15).

Obviously, the solution of (4.6) can be found using the method of "variation of constants". Hence, we get

$$
w(t, x, y)=w_{1}(x) e^{-(a(y)+\gamma(y)) t}+\gamma(y) \int_{0}^{t} e^{-(a(y)+\gamma(y))(t-s)} g(v(s, x)) d s,
$$

or, using the convolution notation

$$
w(t, x, y)=w_{1}(x) e^{-(a(y)+\gamma(y)) t}+\gamma(y) r(\cdot, y) \star g(v(\cdot, x))(t),
$$

with

$$
r(\tau, y)=e^{-(a(y)+\gamma(y)) \tau} .
$$

Moreover, let us notice that (4.5)-(4.8) imply that $v(t, x)$ satisfies the following equation

$$
\frac{\partial v}{\partial t}(t, x)-D \sum_{i, j=1}^{n} q_{i j} \frac{\partial^{2} v}{\partial x_{i} \partial x_{j}}(t, x)+\bar{F}_{0}(t, x)=h(t, x), \quad t>0, x \in \Omega,
$$


with

$$
\bar{F}_{0}(t, x)=\frac{1}{\left|Y^{\star}\right|} \int_{\partial F}\left\{\gamma(y)\left[g(v(t, x))-w_{1}(x) e^{-(a(y)+\gamma(y)) t}-\gamma(y) r(\cdot, y) \star g(v(\cdot, x))(t)\right]\right\} d \sigma .
$$

Proof. We shall not go into the details of the proof of this theorem, since it follows exactly the same steps in the proof of Theorem 2.5. The only difference we have to tackle is the way we treat the coefficients $a^{\varepsilon}$ and $\gamma^{\varepsilon}$. To get rid of the difficulties coming from the fact that they are rapidly oscillating let us notice that in fact they are both uniformly bounded in $L^{\infty}(\Omega)$ and converge strongly therein.

Remark 4.2 The above adsorption model can be slightly generalized by allowing surface diffusion on $S^{\varepsilon}$. This implies that the first equation in (4.3) has to be replaced by

$$
\frac{\partial w^{\varepsilon}}{\partial t}(t, x)-\varepsilon^{2} E \Delta^{\varepsilon} w^{\varepsilon}(t, x)+a^{\varepsilon}(x) w^{\varepsilon}(t, x)=f^{\varepsilon}(t, x) \quad x \in S^{\varepsilon}, t>0,
$$

where $E>0$ is the diffusion constant on the surface $S^{\varepsilon}$ and $\Delta^{\varepsilon}$ is the Laplace-Beltrami operator on $S^{\varepsilon}$ (see Section 2.1.2).

In this case, the homogenized limit is almost the same as before, the only difference being that now, instead of (4.6), we get the following local partial differential equation:

$$
\frac{\partial w}{\partial t}(t, x, y)-E \Delta_{y}^{\partial F} w(t, x, y)+(a(y)+\gamma(y)) w(t, x, y)=\gamma(y) g(v(t, x)), t>0, x \in \Omega, y \in \partial F
$$

where $\Delta^{\partial F}$ denotes the Laplace-Beltrami operator on $\partial F$ and the subscript $y$ indicates the fact that the derivatives are taken with respect to the local variable $y$.

Problems containing a superficial diffusion arise also in the context of climatology: see, for instance, [6] and [17].

The macroscopic behavior of these more general models appeals to some comments that we gather together below.

First of all, it is worth noticing that the bulk behavior of system $\left(\bar{V}^{\varepsilon}\right)-\left(\bar{W}^{\varepsilon}\right)$ involves an additional microvariable $y$. This local phenomena yields a more complicated microstructure of the effective medium; one can say that in equations (4.5)-(4.6) $x$ plays the role of a macroscopic variable, whereas $y$ is a microscopic one.

Secondly, let us observe that the zero-order term in (4.9), namely $\bar{F}_{0}$ involves the convolution $\gamma r \star g$, which shows that we clearly have a memory term in the principal part of our diffusion-reaction equation (4.9).

Acknowledgments. This work has been partially supported by Fondap through its Programme on Mathematical Mechanics. The first author gratefully acknowledges the Chilean and French Governments through the Scientific Committee Ecos-Conicyt. The research of J.I. Díaz was partially supported by project REN2000-0766 of the DGES (Spain) and the RTN HPRN-CT-2002-00274 of the EC. The third author wishes to thank Centro de Modelamiento Matemático de la Universidad de Chile for the warm hospitality and support. 


\section{References}

[1] S.N. Antontsev, A.V. Kazhikhov and V.N. Monakhov, Boundary Value Problems in Mechanics of Nonhomogeneous Fluids, North-Holland, Amsterdam, 1990.

[2] R. ARIs, The Mathematical Theory of Diffusion and Reaction in Permeable Catalysis, Clarendon Press, Oxford, 1975.

[3] V. Barbu, Nonlinear Semigroups and Differential Equations in Banach Spaces, Editura Academiei, Bucuresti, Romania and Noordhoff International Publishing, Leyden, Netherlands, 1976.

[4] J. Bear, Dynamics of Fluids in Porous Media, Elsevier, New York, 1972.

[5] I. Bejenaru, J. I. Díaz And I. I. VRabie, An abstract approximate controllability result and applications to elliptic and parabolic systems with dynamic boundary conditions, Elec. J. Diff. Eqns., 48 (2001), pp. 1-19.

[6] W. H. Berger, S. Burker And E. Vincent, Glacial-holocene transition: climate pulsations and sporadic shutdown of NADW production in abrupt climatic change. Evidence and implications, (W. H. Berger and L. D. Labeyrie eds.), Reidel Publishing Co., Dordrecht, Holland, 1987.

[7] H. BrÉzis, Problèmes unilatéraux, J. Math. Pures et Appl. 51 (1972), pp. 1-168.

[8] D. Cioranescu And P. Donato, Homogénéisation du problème de Neumann non homogène dans des ouverts perforés, Asymptotic Anal. 1 (1988), pp. 115-138.

[9] D. Cioranescu, P. Donato and H. Ene, Homogenization of the Stokes problem with non homogeneous slip boundary conditions, Mathematical Methods in Applied Sciences, 19 (1996), pp. 857-881.

[10] D. Cioranescu and J. Saint Jean Paulin, Homogenization in open sets with holes, J. Math. Anal. Appl. 71 (1979), pp. 590-607.

[11] C. Conca, On the application of the homogenization theory to a class of problems arising in fluid mechanics, J. Math. Pures Appl. 64 (1985), pp. 31-75.

[12] C. Conca and P. Donato, Non homogeneous Neumann problems in domains with small holes, RAIRO Modél. Math. Anal. Numér. 22 (1988), pp. 561-607.

[13] C. Conca, J. I. Díaz, A. Liñán and C. Timofte, Homogenization in chemical reactive flows through porous media, Preprint CMM, University of Chile (2002).

[14] J. I. DíAz, Nonlinear Parabolic Problems in Fluid Mechanics, Lecture Notes of a Postgraduate Course at the Universidad de Oviedo (Spain), notes in Spanish taken by A. Mateos and R. Sarandeses, 1992.

[15] J. I. Díaz, Two Problems in Homogenization of Porous Media, Extracta Mathematica, 14 (1999), pp. 141-155.

[16] J. I. DíAz And I. StAKgold, Mathematical aspects of the combustion of a solid by distributed isothermal gas reaction, SIAM J. Math. Anal., 26, 2 (1994), pp. 305-328. 
[17] J. I. DíAz And L. Tello, Sobre un modelo de balance de energía superficial acoplado con un océano profundo, CD-Rom Actas XVII CEDYA / VII CMA, (L. Ferragut and A. Santos eds.), Servicio de Publicaciones de la Universidad de Salamanca, 2001.

[18] U. Hornung, Homogenization and Porous Media, Springer, New York, 1997.

[19] U. Hornung AND W. JäGER, Diffusion, convection, adsorption and reaction of chemicals in porous media, J. Diff. Eqns. 92 (1991), pp. 199-225.

[20] J.L. Lions, Quelques Méthodes de Résolution des Problèmes aux Limites non Linéaires, Dunod, Gauthier-Villars, Paris, 1969.

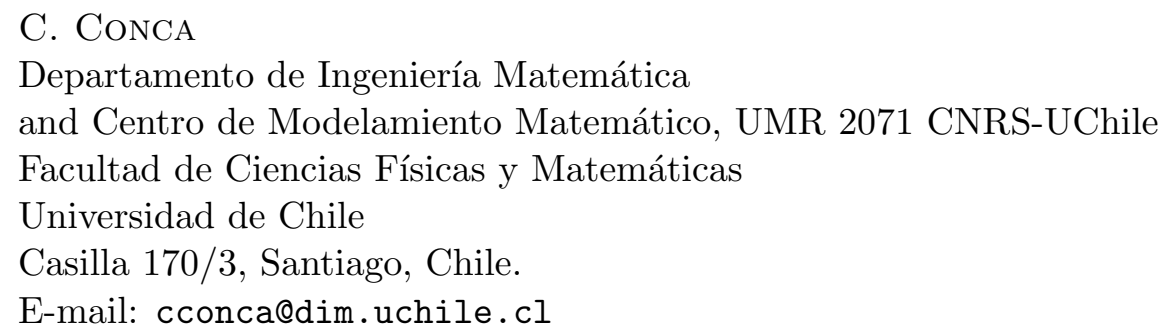

J.I. DÍAZ

Departamento de Matemática Aplicada

Facultad de Matemáticas

Universidad Complutense

28040 Madrid, Spain.

E-mail : jidiaz@ucm.es

C. Timofte

Department of Mathematics

Faculty of Physics

University of Bucharest

P.O. Box MG-11, Bucharest-Magurele, Romania.

E-mail: claudiatimofte@hotmail.com 